\title{
Multiple Septic Emboli in the Skin, Lungs, and Brain due to Corynebacterium Striatum Bacteremia in a Patient with Acute Myelogenous Leukemia
}

\author{
Motoharu Shibusawa*, Jiro Tadokoro, Shouhei Sato, Makoto Kashimura \\ Shinmatsudo Central General Hospital, Department of Hematology \\ *Corresponding author: m_sibusawa@hotmail.com
}

Received November 04, 2018; Revised December 06, 2018; Accepted December 28, 2018

\begin{abstract}
A 29-year-old man developed fever after chemotherapy for acute myelogenous leukemia. Corynebacterium striatum was detected via blood culture, and antibacterial treatment with vancomycin started. In the course of treatment, papules of the skin, multiple nodular shadows of the lung, cerebral infarction, and subdural empyema appeared. A diagnosis of multiple septic emboli in the skin, lungs, and brain due to C. striatum bacteremia was made from the clinical course. The antibacterial treatment improved skin and pulmonary lesions. The subdural empyema was considered as a postoperative infection of burr-hole craniotomy. It improved by performing craniotomy twice with drainage and empiric antibiotic therapy. In our case, neutropenia was present as an immunodeficiency, which made bacteremia severe. There has not been reported a case of septic emboli of three lesions; skin, lung, and brain. C. striatum bacteremia may result in septic emboli and should immediately be treated with antibiotics.
\end{abstract}

Keywords: Corynebacterium striatum, acute myelogenous leukemia, septic emboli

Cite This Article: Motoharu Shibusawa, Jiro Tadokoro, Shouhei Sato, and Makoto Kashimura, "Multiple Septic Emboli in the Skin, Lungs, and Brain due to Corynebacterium Striatum Bacteremia in a Patient with Acute Myelogenous Leukemia." American Journal of Medical Case Reports, vol. 6, no. 12 (2018): 241-246. doi: 10.12691/ajmcr-6-12-4.

\section{Introduction}

Corynebacterium striatum is a bacterium that causes bloodstream infection. We experienced bloodstream infection of C. striatum, resulting in multiple septic emboli in the skin, lungs, and brain in a patient with acute myelogenous leukemia (AML).

\section{Case Presentation}

A 29-year-old man was presented with purpura and admitted to our hospital. He had nine tooth caries. His body temperature was $37.4^{\circ} \mathrm{C}$, blood pressure was $127 / 75$ $\mathrm{mmHg}$, pulse rate was 66 beats per minute, and oxygen saturation was $99 \%$ in ambient air. He had petechiae in the oral mucosa and on the bilateral lower legs. He had no palpable lymphadenopathy on the cervical, axillary, and inguinal region. Chest auscultation revealed no rales or murmur. The abdomen was soft, without distention, rebound tenderness, guarding and palpable hepatosplenomegaly. His laboratory test results (Table 1) revealed hyperleukocytosis (white blood cell count, $218 \times 10^{9}$ cells/L; differential count: blasts, $98 \%$; neutrophils, $0.5 \%$; lymphocytes, $1.5 \%$ ). thrombocytopenia (platelet count $24 \times 10^{9} / \mathrm{L}$ ), normal hemoglobin concentration (hemoglobin level $17.1 \mathrm{~g} / \mathrm{dL}$ ), elevated lactate dehydrogenase (523 IU/L), prolonged prothrombin time $(17.5 \mathrm{sec})$, normal activated partial thromboplastin time $(27.6 \mathrm{sec})$, low fibrinogen level (96 $\mathrm{mg} / \mathrm{dL})$, elevated d-dimer $(32.0 \mu \mathrm{g} / \mathrm{mL})$, and fibrin/fibrinogen degradation products $(97.2 \mu \mathrm{g} / \mathrm{ml})$. The bone marrow examination was performed and resulted in making a diagnosis of AML. Figure 1 shows the patient's clinical course. On the first day of admission, empiric antibiotic therapy was initiated by administering $2 \mathrm{~g}$ cefepime (CFPM) every 12 hours. Cytoreduction with $2000 \mathrm{mg}$ hydroxyurea of daily and an administration of thrombomodulin alfa for 14 days and transfusions of fresh frozen plasma for disseminated intravascular coagulation (DIC) were started. Anemia and thrombocytopenia due to leukemia and chemotherapy were treated with red cell and platelet transfusions to maintain the hemoglobin level at $7.0 \mathrm{~g} / \mathrm{dL}$, platelet count at $20 \times 10^{9} / \mathrm{L}$, respectively. Although the dose of hydroxyurea was gradually increased up to $5000 \mathrm{mg}$ daily, it failed in cytoreduction. On day 11 , a peripherally inserted central catheter (PICC) was inserted into the left cubital fossa for intravenous administration of antineoplastic agents, followed by the initiation of chemotherapy (cytarabine, $100 \mathrm{mg} / \mathrm{m}^{2}$ day 11-17; idarubicin, $12 \mathrm{mg} / \mathrm{m}^{2}$ day15-17). Chemotherapy decreased the neutrophil and blast count, and lysed leukemia cells, resulting in exacerbation of DIC. On day 
18, we removed the PICC because of completing the administration of antineoplastic agents. On day 22, his body temperature was $40.8^{\circ} \mathrm{C}$, blood pressure was $137 / 75$ $\mathrm{mmHg}$, pulse rate was 77 beats per minute, and oxygen saturation was $96 \%$ in ambient air, his consciousness was clear. The neutrophil count on this day was $0 / \mathrm{L}$. We obtained two sets of blood cultures. After approximately 48 hour incubation, the microbial growth was detected in two aerobic blood-culture bottles on day 24. The Gram staining of the blood-culture revealed Gram-positive rods, and we started empiric antibiotic therapy with vancomycin (VCM) with therapeutic drug monitoring. The trough values of VCM were $4.7-21.4 \mu \mathrm{g} / \mathrm{mL}$, and the mean value was $10.4 \mu \mathrm{g} / \mathrm{mL}$. In addition, a daily administration of $5 \mathrm{~g} \gamma$-immunoglobulin for 3 days was initiated. The isolated bacteria were recognized as C. striatum, which is susceptible to VCM (Table 2). On day 25, Papules, ranging 3-5 $\mathrm{mm}$ in diameter, appeared on both forearms and lower legs (Figure 2). On day 29, disturbance of consciousness (Glasgow coma scale (GCS) score 7: E1V2M4) and convulsions twisting the neck and moving extremities developed. A head computed tomography (CT) scan revealed a low-density area in the left subdural space, which was consistent with a subdural hematoma secondary to thrombocytopenia. We initiated sedation with propofol because of frequent convulsions. The bone marrow examination revealed hematological remission of AML, and $100 \mathrm{mg} / \mathrm{m}^{2}$ of filgrastim was started for 4 days. Subsequently, neutrophil count increased. On day 30, we performed a burr-hole craniotomy with drainage and drained the hematoma. The neutrophil count on this day was $0.077 \times 10^{9} / \mathrm{L}$, suggesting a high risk of the postoperative infection. On day 31 , a whole-body CT scan revealed multiple nodular shadows, ranged 3-26 $\mathrm{mm}$ in diameter appeared, in the bilateral lungs (Figure 3). The patient became bedridden by severe bacteremia and could not intake by disturbance of consciousness. On day 34, PICC was inserted in the right cubital fossa for total parenteral nutrition because two bottles of blood-culture obtained on day 27 exhibited no microbial growth for 7 days. On day 37, a head magnetic resonance imaging (MRI) scan of diffusion-weighted, T2-weighted, and fluid-attenuated inversion recovery sequence revealed a high-intensity area in the left temporal lobe (Figure 4). A diagnosis of acute-phase cerebral infarction was made by this result. Papules of the skin, multiple nodular shadows of the lung, and cerebral infarction were consistent with septic emboli. A diagnosis of multiple septic emboli in the skin, lungs, and brain due to C. striatum bacteremia was made by the course. On day 39, disturbance of consciousness improved; GCS score 10: E4V1M5, but aphasia developed as a complication of cerebral infarction. On day 43, the operation wound of burr-hole craniotomy discharged pus. A head CT scan revealed a low-density area in the left subdural space, indicating a subdural empyema. Empiric antibiotic therapy with VCM, meropenem $1 \mathrm{~g}$ every 8 hours, and doripenem $1 \mathrm{~g}$ every 8 hours was performed for the following causative pathogens of infection after penetrating neurosurgery: Staphylococcus aureus, S. epidermidis, and Streptococcus species (anaerobic and aerobic), Enterobacteriaceae, Clostridium species [1]. On day 44, we performed burr-hole craniotomy with drainage for draining the abscess. Gram staining of the abscess failed to show bacteria, and the isolation culture (aerobe, anaerobe, S. aureus, S. species, and Mycobacterium) exhibited no microbial growth. On day 45, the patient started rehabilitation and on day 47, transthoracic echocardiography revealed no vegetation and the patient removed the PICC by himself because of delirium. From day 51 , the patient was able to intake. On day 52, a result of T1-weighted sequence MRI scan of the head with intravenous contrast material revealed disseminated hypointense with ringed-shaped enhancement primarily in the left subdural space (below the operation wound) (Figure 5), and outside of the left cerebral hemisphere and on the left tentorium cerebelli, which was consistent with the remaining subdural empyema. On day 53, burr-hole craniotomy with subdural empyema drainage was performed again to drain the abscess. Gram staining of the abscess failed to show a bacteria and the isolation culture (aerobe, anaerobe, Staphylococcus species, S. aureus, Pseudomonas aeruginosa, Fungi, Listeria species, and acid-fast bacterium) exhibited no microbial growth. Polymerase chain reaction of Tuberculosis, Mycobacterium avium, and Mycobacterium intracellulare were negative. On day 67, we started chemotherapy (cytarabine, $12 \mathrm{mg} / \mathrm{m}^{2}$ day $67-80+$ aclarubicin, $12 \mathrm{mg} / \mathrm{m}^{2}$ day 67-70). A T1-weighted sequence MRI scan of the head with intravenous contrast material on days 65 and 89 revealed reduced but remaining abscess. The patient was discharged on day 91 and readmitted after 2 days. Subsequently, two courses of the chemotherapies with the empiric antibiotic therapy were performed. A T1-weighted sequence MRI scan of the head with intravenous contrast material on day 117 after the first admission revealed a reduced but remaining abscess.

Table 1. Laboratory data on admission

\begin{tabular}{|c|c|c|c|c|c|c|c|c|}
\hline White blood cells & $218 \times 10^{9}$ & $/ \mathrm{L}$ & Total protein & 8.6 & $\mathrm{~g} / \mathrm{dL}$ & Natrium & 141 & $\mathrm{mEq} / \mathrm{L}$ \\
\hline Neutrophils & 0.5 & $\%$ & Albumin & 5.8 & $\mathrm{~g} / \mathrm{dL}$ & Kalium & 2.8 & $\mathrm{mEq} / \mathrm{L}$ \\
\hline Lymphocyte & 1.5 & $\%$ & Aspartate aminotransferase & 36 & $\mathrm{IU} / \mathrm{L}$ & Chlorine & 97 & $\mathrm{mEq} / \mathrm{L}$ \\
\hline Blast & 98 & $\%$ & Alanine aminotransferase & 60 & $\mathrm{IU} / \mathrm{L}$ & Calcium & 10.7 & $\mathrm{mg} / \mathrm{dL}$ \\
\hline Red blood cell & $537 \times 10^{10}$ & $/ \mathrm{L}$ & Lactate dehydrogenase & 523 & $\mathrm{IU} / \mathrm{L}$ & C-reactive protein & 1.74 & $\mathrm{mg} / \mathrm{dL}$ \\
\hline Hemoglobin level & 17.1 & $\mathrm{~g} / \mathrm{dL}$ & Total bilirubin & 0.6 & $\mathrm{mg} / \mathrm{dL}$ & Blood sugar & 93 & $\mathrm{mg} / \mathrm{dL}$ \\
\hline Platelet & $24 \times 10^{9}$ & $/ \mathrm{L}$ & Direct bilirubin & 0.1 & $\mathrm{mg} / \mathrm{dL}$ & & & \\
\hline Prothrombin time & 17.5 & sec & Alkaline phosphatase & 308 & IU/L & & & \\
\hline Activated partial thromboplastin time & 27.6 & sec & Blood urea nitrogen & 10 & $\mathrm{mg} / \mathrm{dL}$ & & & \\
\hline Fibrinogen & 96 & $\mathrm{mg} / \mathrm{dL}$ & Creatine kinase & 0.87 & $\mathrm{mg} / \mathrm{dL}$ & & & \\
\hline D-dimer & 32 & $\mu \mathrm{g} / \mathrm{mL}$ & Uric acid & 9.4 & $\mathrm{mg} / \mathrm{dL}$ & & & \\
\hline Fibrin/fibrinogen degradation products & 97.2 & $\mu \mathrm{g} / \mathrm{mL}$ & & & & & & \\
\hline
\end{tabular}




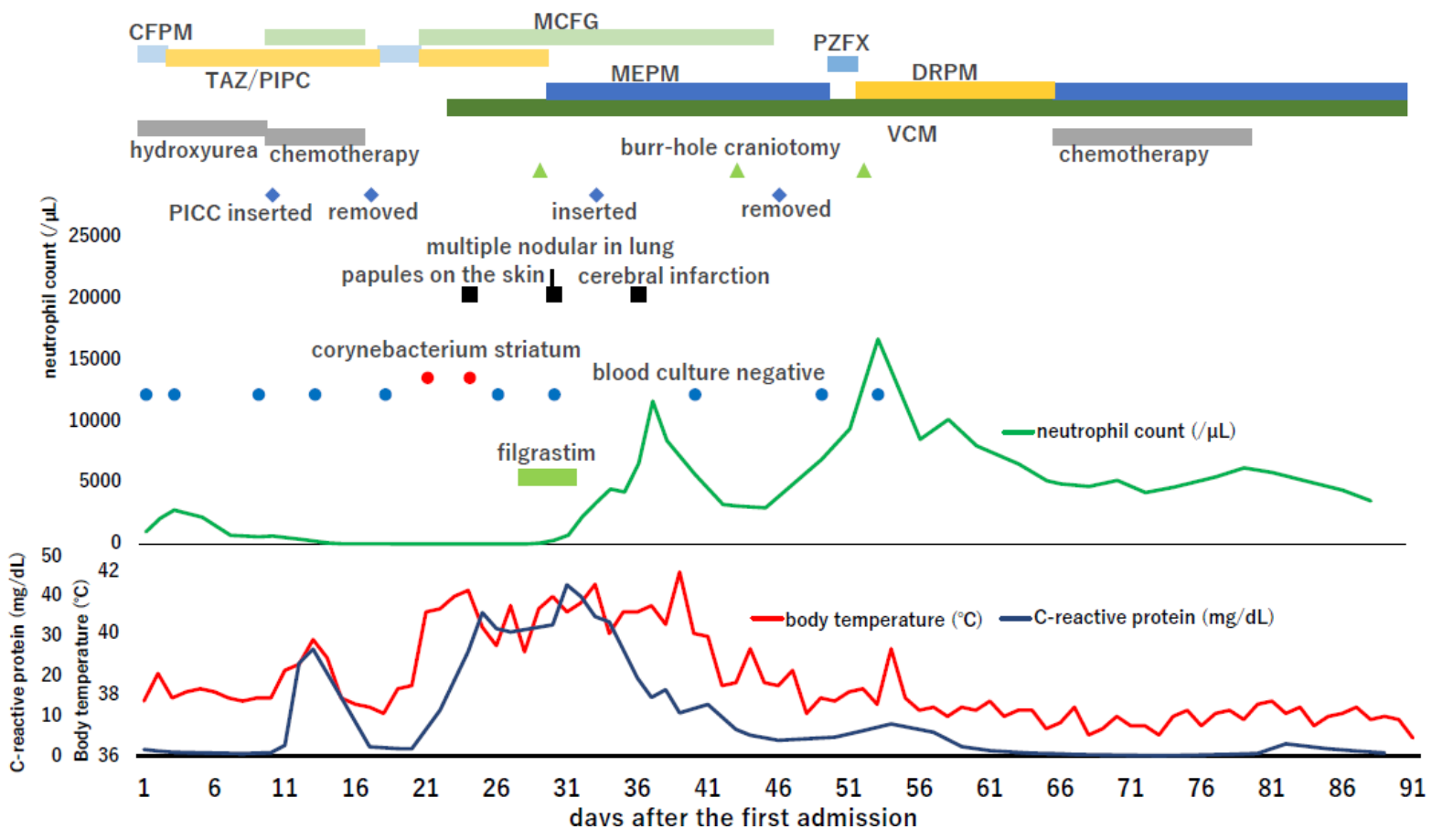

Chemotherapy decreased the neutrophil and blast count. Blood culture on days 22 and 25 revealed the microbial growth of Corynebacterium striatum. Papules on the skin appeared on day 25. Multiple nodules in a lung CT on day 31 and high-intensity area in the left temporal lobe in a head MRI scan on day 37 appeared, respectively. As the bacteremia was treated, fever relieved and C-reactive protein decreased.

The dose and abbreviation of antibiotics and antifungal agent cefepime (CFPM): $2 \mathrm{~g}$ every 12 hours, tazobactam/piperacillin (TAZ/PIPC): $4.5 \mathrm{~g}$ every 8 hours, micafungin (MCFG): $150 \mathrm{mg}$ every 24 hours, vancomycin (VCM), meropenem (MEPM): $1 \mathrm{~g}$ every 8 hours, pazufloxacin (PZFX): $1 \mathrm{~g}$ every 12 hours, doripenem (DRPM) $1 \mathrm{~g}$ every 8 hours

Figure 1. Clinical course of our case

(a)

(b)

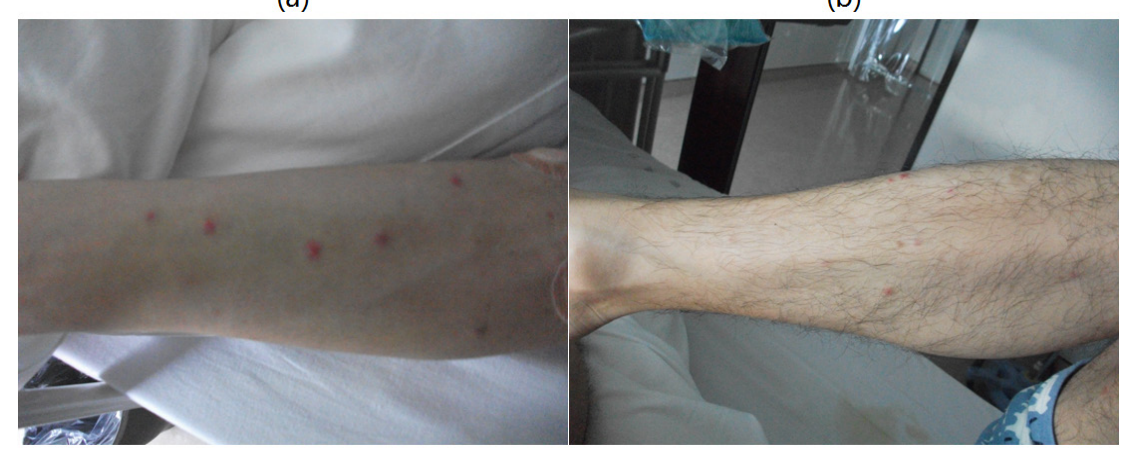

Figure 2. Skin lesion on day 25 Papules ranging 3-5 mm in diameter appeared: (a) on both forearms and (b) on both lower legs.

Table 2. Susceptibility to antibiotics of the cultured Corynebacterium striatum $S=$ sensitive, $R=$ resistance

\begin{tabular}{|l|l|}
\hline benzylpenicillin (PCG) & $\mathrm{R}$ \\
\hline ampicillin (ABPG) & $\mathrm{R}$ \\
\hline sulperazon (SBT/CPZ) & $\mathrm{R}$ \\
\hline cefazolin (CEZ) & $\mathrm{R}$ \\
\hline cefotiam (CTM) & $\mathrm{R}$ \\
\hline flomoxef (FMOX) & $\mathrm{R}$ \\
\hline cefepime (CFPM) & $\mathrm{R}$ \\
\hline meropenem (MEPM) & $\mathrm{R}$ \\
\hline gentamicin (GM) & $\mathrm{S}$ \\
\hline erythromycin (EM) & $\mathrm{R}$ \\
\hline clindamycin (CLDM) & $\mathrm{R}$ \\
\hline minocycline (MINO) & $\mathrm{S}$ \\
\hline levofloxacin (LVFX) & $\mathrm{R}$ \\
\hline vancomycin (VCM) & $\mathrm{S}$ \\
\hline fosfomycin (FOM) & $\mathrm{R}$ \\
\hline
\end{tabular}

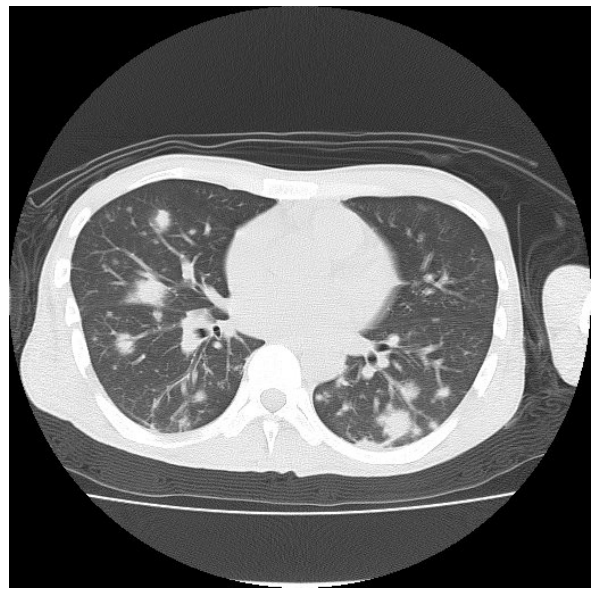

Multiple nodular shadows ranging 3-26 $\mathrm{mm}$ in diameter appeared on the bilateral lungs. Some nodules were located in the subpleural, suggesting that their pathology is associated with the blood flow.

Figure 3. Chest $\mathrm{CT}$ on day 31 
(a)

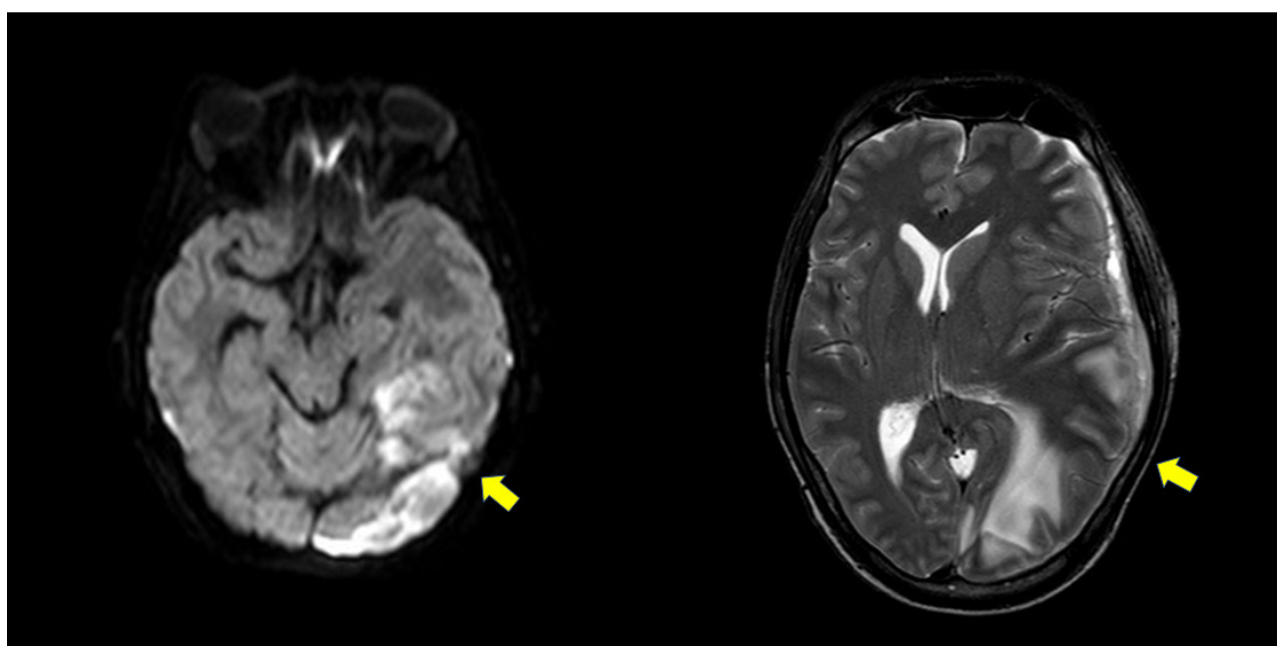

Figure 4. MRI scan of the head on day 37: (a), diffusion-weighted sequence; a high-intensity area in the left temporal lobe; (b), T2-weighted sequence; in the left cerebral hemisphere, the cerebral sulci were narrow, and the lateral ventricle was compressed, indicating swelling of the left cerebral hemisphere

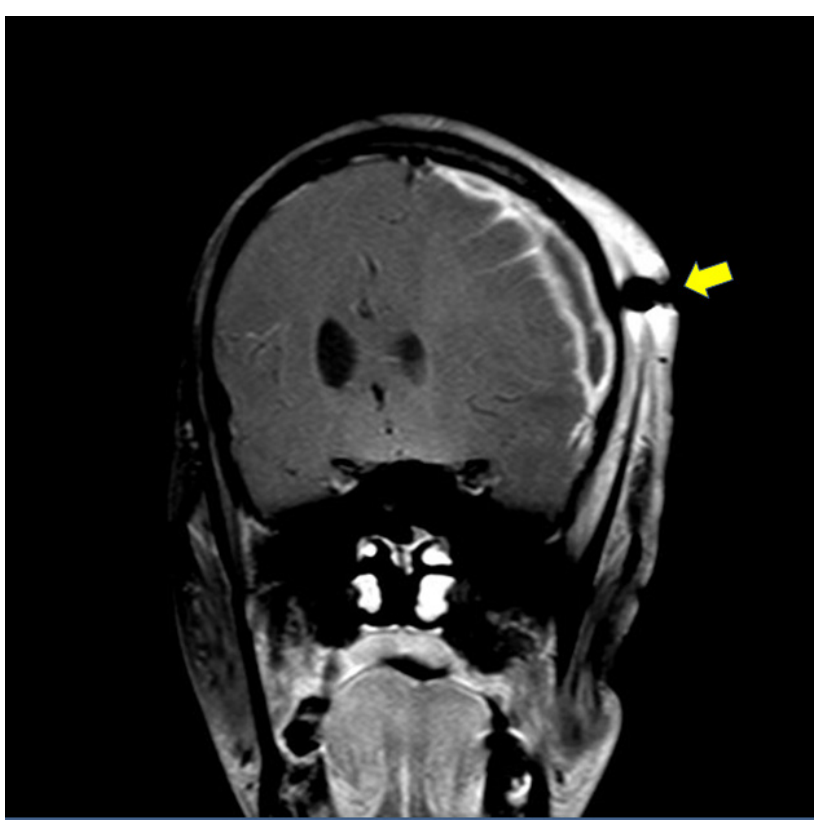

Figure 5. T1-weighted sequence MRI scan of the head with intravenous contrast material on day 52 disseminated hypointense with ringed-shaped enhancement primarily in the left subdural space (below the operation wound)

As the bacteremia was treated, fever relieved and C-reactive protein decreased. Papules on the skin and nodules on the lung resolved. The disturbance of consciousness was considered as secondary to severe bacteremia, subdural hematoma, and cerebral infarction also gradually resolved. Craniotomy performed twice with drainage and the empiric therapy improved the subdural empyema. On day 143 after the first admission, the patient was transferred to another hospital for hematopoietic stem cell transplantation.

\section{Outcome and Follow up}

Two-course chemotherapy (consolidation therapies) and hematopoietic stem cell transplantation from an unrelated donor was performed with empiric antibiotic therapy consist of CFPM: 2 g every 12 hours and VCM.
Three months later hematopoietic stem cell transplantation, a MRI scan of the head with intravenous contrast material revealed no subdural empyema.

\section{Discussion}

C. striatum ubiquitously colonizes the skin and mucous membranes and primarily infects patients with immunosuppression or indwelling devices. Coryneform bacteria are irregularly shaped, non-spore forming, aerobic, and Gram-positive rod. Reportedly, main infection sites of C. striatum are intravenous catheter-related bloodstream infection, native and prosthetic valve endocarditis, pacemaker-related endocarditis, meningitis, pulmonary abscess, septic arthritis, and vertebral osteomyelitis. C. striatum is susceptible to VCM, teicoplanin, and linezolid [2]. In this case, neutropenia was present as immunodeficiency, which made the bacteremia severe. The bacteremia was unlikely to be a catheter-associated infection because the PICC had been removed at its onset. C. striatum was susceptible to VCM. The administration of VCM and an increase in the neutrophil count improved the bacteremia. In the course of treatment, papules of the skin, multiple nodular shadows of the lung, and cerebral infarction appeared and they were diagnosed as multiple septic emboli due to C. striatum bacteremia. Infective endocarditis was considered as an etiologies of the multiple septic emboli. Ye, R. et, al reviewed 168 cases with septic pulmonary emboli. 107 cases were evaluated by echocardiography (ECG) (only transthoracic ECG; 82 cases, only transesophageal ECG; 44 cases, and both 19 case). ECG revealed vegetations in 52 cases (48.6\%), including vegetations on the tricuspid valve in 45 cases $(86.5 \%)$, vegetations on the pulmonary valve in 2 cases and vegetations on the atrium and endocardium in 5 cases [3]. Our case had pulmonary emboli. Transthoracic echocardiography on day 47 revealed no vegetation and transesophageal echocardiography could not be performed because of disturbance in consciousness. Even though evidence indicating infective endocarditis was not revealed, it might be present. 
Table 3. Cases complicated by septic emboli due to coryneform bacteria bloodstream infection

\begin{tabular}{|c|c|c|c|c|c|}
\hline case & age/sex & pathogen & underlying disease & $\begin{array}{c}\text { central venous catheter } \\
\text { at the onset }\end{array}$ & septic emboli \\
\hline our case & $29 /$ male & $\begin{array}{c}\text { Corynebacterium } \\
\text { striatum }\end{array}$ & $\begin{array}{c}\text { acute myelogenous } \\
\text { leukemia }\end{array}$ & $\begin{array}{c}\text { papules on the skin, } \\
\text { multiple pulmonary nodules, } \\
\text { cerebral infarction }\end{array}$ & absent \\
\hline 1 & $72 /$ female & $\begin{array}{c}\text { Corynebacterium } \\
\text { striatum }\end{array}$ & $\begin{array}{c}\text { coronary disease and } \\
\text { hypertension }\end{array}$ & present & 6 \\
\hline 2 & $18 /$ male & $\begin{array}{c}\text { Corynebacterium } \\
\text { jeikeium }\end{array}$ & $\begin{array}{c}\text { acute myelogenous } \\
\text { leukemia }\end{array}$ & 7 \\
\hline
\end{tabular}

In our case, septic emboli appeared in a different phase. The cutaneous lesions appeared in a phase of low in neutrophil count, while, the pulmonary and cerebral lesion developed in a phase of a sudden increase in the neutrophil count. This difference might depend on etiology of septic emboli. The cutaneous lesion was considered as vasculopathy due to septic emboli. Delgado-Jimenez, Y. et, al investigated 32 cases of acute bacterial septic vasculopathy. The most common skin signs were purpuric lesions, petechiae followed by vesicles, bullae, pustules, distal ischemia, and nodules. Cutaneous lesions developed simultaneously or within the first 24 hours after onset of sepsis (56.3\%), followed by within first seven days (34.4\%), and more than 7 days $(9.4 \%)$. Skin biopsies showed thrombi in $100 \%$ of cases. Other common findings were inflammatory infiltrate, blood extravasation, and epidermal involvement. Bacteria within the vascular wall were seen in $21.9 \%$ of cases and fibrinoid necrosis in $25 \%$ [4]. In our case, the cutaneous lesions were papules and developed 4 days after onset of bacteremia at neutropenic period. They might be formed by vasculopathy due to septic emboli. On the other hand, the pulmonary emboli and cerebral infarction appeared 10 and 16 days after onset of the bacteremia respectively, when the neutrophil count suddenly increased. It is presumed that the increase in neutrophil count starts the phagocytosis of bacteria and colonization of the neutrophil, subsequently, followed by inflammation. These may result in remarkable findings of septic emboli.

Previous study reported predictive factors for mortality in coryneform bacteria bloodstream infection in hematological patients. As the predictive factors, infection by more than two pathogens, renal impairment, and absence of a central venous catheter at the onset of bloodstream infection have been identified [5]. In our case, the predictive factors was absence of a central venous catheter at the onset of bloodstream infection.

Furthermore, cases complicated by septic emboli due to coryneform bacteria bloodstream infection have been reported [6,7], (Table 3). Like our case, cases 1 and 2 had septic emboli in the lungs but not in the brain and skin. Especially, same to our case, case 2 was a case of AML treated with chemotherapy and neutropenia was present at the onset of bacteremia. Multiple pulmonary nodules on chest X-ray and CT scan appeared ten days after onset of the bacteremia when the neutrophil count increased. This course is same as our case. The characteristic point of our case was septic emboli were present not only in the lungs but also the skin and brain. There has not been reported a case of septic emboli of three lesions; skin, lung, and brain.

On the other hand, our case was accompanied by a subdural empyema. The central nervous system is primarily contaminated thought the following three routes: hematogenic, via adjacency, and neural [8]. The most common predisposing condition in subdural empyema is an otorhinolaryngologic infection, especially paranasal sinus. Another predisposing condition is neurosurgical procedures, the infection of a preexisting subdural hematoma, and skull trauma. Metastatic infection is principally from pulmonary sources [9]. In our case, the subdural empyema developed after burr-hole craniotomy with hematoma drainage, which was performed under the condition of the high risk of postoperative infection. The subdural empyema primarily existed below the operation wound. However, microbial examinations of the drained abscess did not identify a causative pathogen, and the subdural empyema was possibly a postoperative infection or an infection of a preexisting subdural hematoma. C. striatum bacteremia was unlikely to cause the subdural empyema hematogenous, because, at the onset of the subdural abscess, blood-cultures exhibited no microbial growth.

In conclusion, C. striatum bacteremia may result in septic emboli and should immediately be treated with antibiotics.

\section{Acknowledgements}

We appreciate for Dr. Yoshihiko Uemura for performing burr-hole craniotomy for our case.

\section{Statement of Competing Interests}

Authors have no competing interest to declare.

\section{List of Abbreviations}

Corynebacterium striatum: C. striatum; acute myelogenous leukemia: AML; disseminated intravascular coagulation: DIC; peripherally inserted central catheter: PICC; Glasgow coma scale: GCS; computed tomography: CT; magnetic resonance imaging: MRI; cefepime: CFPM; tazobactam/piperacillin: TAZ/PIPC; micafungin: MCFG; vancomycin: VCM; meropenem: MEPM; pazufloxacin: PZFX; doripenem: DRPM.

\section{References}

[1] Brouwer, M. C., Tunkel, A. R., McKhann, G. M., 2nd and van de Beek, D., "Brain abscess". N Engl J Med, 371(5). 447-56, Jul 31 2014.

[2] Rose, Kim and Annette C, Reboli, Other Coryneform Bacteria and Rhodococci., in Mandell, Douglas, and Bennett's principles and practice of infectious disease, Elsevier, Philadelphia, 2015, 2373-2376. 
[3] Ye, R., Zhao, L., Wang, C., Wu, X. and Yan, H., "Clinical characteristics of septic pulmonary embolism in adults: a systematic review". Respir Med, 108(1). 1-8, Jan 2014.

[4] Delgado-Jimenez, Y., Fraga, J., Requena, C., Requena, L., Aragues, M., Fernandez Herrera, J. and Garcia Diez, A., "Acute bacterial septic vasculopathy". Int J Dermatol, 52(9). 1071-80, Sep 2013.

[5] Kimura, S. I., Gomyo, A., Hayakawa, J., Akahoshi, Y., Harada, N., Ugai, T., Komiya, Y., Kameda, K., Wada, H., Ishihara, Y., Kawamura, K., Sakamoto, K., Sato, M., Terasako-Saito, K., Kikuchi, M., Nakasone, H., Kanda, J., Kako, S., Tanihara, A. and Kanda, Y., "Clinical characteristics and predictive factors for mortality in coryneform bacteria bloodstream infection in hematological patients". J Infect Chemother, 23(3). 148-153, Mar 2017.
[6] Severo, C. B., Guazzelli, L. S., Barra, M. B., Hochhegger, B. and Severo, L. C., "Multiple pulmonary nodules caused by Corynebacterium striatum in an immunocompetent patient". Rev Inst Med Trop Sao Paulo, 56(1). 89-91, Jan-Feb 2014.

[7] Sato, K. and Uchiyama, M., "Corynebacterium jeikeium bacteraemia and pulmonary infiltrates in a patient with acute myelogenous leukaemia". BMJ Case Rep, 2012. Mar 202012.

[8] Sarrazin, J. L., Bonneville, F. and Martin-Blondel, G., "Brain infections". Diagn Interv Imaging, 93(6). 473-90, Jun 2012.

[9] Tunkel, Allan R., Subdural Empyema, Epidural Abscess, and Suppurative Intracranial Thrombophlebitis, in Mandell, Douglas, and Bennett's principles and practice of infectious disease, Elsevier, Philadelphia, 2015, 1177-1180 